\title{
Arterial Vascular Variation of the Head and Neck and Its Clinical Significance
}

Shi-kui Shi*

Department of Radiology, The First Affiliated Hospital of Bengbu Medical College, People's Republic of China

*Corresponding author: Shi-kui Shi, Department of Radiology, The First Affiliated Hospital of Bengbu Medical College, China, E-mail: sskuijlp@163.com

Received date: April 25, 2017; Accepted date: August 28, 2017; Published date: August 31, 2017

Copyright: (c) 2017 Shi S. This is an open-access article distributed under the terms of the Creative Commons Attribution License, which permits unrestricted use, distribution, and reproduction in any medium, provided the original author and source are credited.

\section{Commentary}

Arterial vascular variation of the head and neck is common and is often overlooked because of the lack of obvious clinical symptoms. Variations are generally detected accidentally by head and neck computed tomography angiography (CTA), magnetic resonance angiography (MRA) or ultrasound. Considering the lack of adequate understanding of the presence and location of the arterial vascular variations of the head and neck, vessels are easily injured or incorrectly ligated intraoperatively. Sometimes this could even cause fatal consequences. Therefore, in recent years, increasing attention has been paid to the diagnosis and assessment of arterial vascular variations of the head and neck.

As an important part of the brain's blood supply system, there are many types of variations in the vertebral artery. Common variations of vertebral arteries mainly include variations in origin, in number, in the first transverse foramen during the ascending process, and in diameter, amongst others. A variation in origin refers to the origin of a vertebral artery from an artery other than the subclavian artery. A left vertebral artery originating from the aortic arch between the left common carotid artery (CCA) and the left subclavian artery is the most common variation in the origin of the vertebral artery. Although the blood supply is not majorly affected, the vertebral artery blood pressure often increases significantly. This can lead to vertebral artery atherosclerosis, stenosis or obstruction. Increased pressure within the aortic arch further becomes one of the prime reasons for rupture or formation of arterial dissection of the left vertebral artery. A vertebral artery originating from the CCA is rare; however, researchers have found that the aberrant right subclavian artery and origin variation of the right vertebral artery have a certain correlation [1]. A vertebral artery of anomalous origin often leads through the first transverse foramen higher than the normal origin, leading to the initial segment of the vertebral artery being extensive and tortuous and a lack of protection of the cervical transverse process. Rotation of the neck may cause vertebral artery spasms or dissection and even occlusion [2]. The vertebral artery of anomalous origin generally leads through the fifth, the fourth, or even the seventh vertebra other than the sixth vertebra. The neurosurgeon should completely understand and evaluate the presence of vertebral artery variations to avoid damage to the vertebral artery and the blood supply to the spinal cord and brain during surgery. A variation in number of vertebral arteries means unilateral or bilateral absence of vertebral artery or the increase of the number of vertebral arteries. Hypoplasia of vertebral artery is common. Vertebral artery hypoplasia refers to that the diameter of vertebral artery is less than $2.5 \mathrm{~mm}$ or the diameter of one side vertebral artery is less than $50 \%$ of the contralateral side. But the diagnostic criteria of vertebral artery hypoplasia are not uniform. Chaturvedi et al. [3] pointed out that the smaller vertebral artery developed atherosclerosis earlier than the normal side, thus further inducing or aggravating vertebrobasilar insufficiency. The variation of the basilar artery dilation and fenestration is common. Sim et al. [4] pointed out that this kind of variation was only random, non-symptomatic, was not clinically relevant. However, Miyazaki et al. [5] believed that there might be some correlation between the basilar artery fenestration with multiple intracranial aneurysms.

A high level of bifurcation variation of the CCA means that the CCA issues the internal carotid artery (ICA) and external carotid artery from the level of the hyoid bone, which generally does not affect normal blood supply. However, the presence of these variations should be considered during maxillofacial surgical repair, hemostasis of facial blood supply, arterial ligation, and others. The ICA variations include a curved course, kinking, coiling or the anomalous origin [6]. And bilateral duplicated ICAs can also be seen, of which an aberrant ICA sometimes presenting as a middle ear mass [7]. Should injury to these variant ICAs occur, the neurosurgeon should follow specific guidelines to avoid potentially serious complications. The common variations of the ascending segment of the anterior cerebral artery (ACA) include the azygos ACA, the triploid ACA, and the fenestration of the ACA. It is generally believed that the incidence of aneurysms at the bifurcation of the distal azygos ACA is higher [8]. The presence of the triploid ACA may make it difficult to expose the accurate location of the ACA, easily damaging the artery during surgery. Fenestration of the ascending segment of the ACA does not produce significant clinical symptoms [9]. The incidence of hypoplasia of the anterior communicating segment of the ACA is approximately $7 \% \sim 10 \%$ and hypoplasia or absence often leads to the dilation of the contralateral vessel.

Variations of the middle cerebral artery (MCA) are not uncommon, which generally include duplicated MCA, accessory MCA, fenestration, early bifurcation and trifurcation. Uchino et al. [10] presented a case of duplicated right MCA that arose from the origin of the right hyperplastic anterior choroidal artery. The fetal origin of the posterior cerebral artery (FPCA) is an important pathway with respect to communication between the ICA and the vertebrobasilar artery, which were usually detected co-existent with vertebral artery hypoplasia [11]. Anatomical variation of FPCA is a risk factor associated with ischemic cerebral infarction. In addition, during the development of human embryos, there are four other temporary pathways connecting the ICA and the basilar artery: persistent trigeminal artery, persistent hypoglossal artery, proatlantal intersegmental artery, and persistent internal auditory artery. If these pathways are not degraded in adults, it is known as persistent cervicalbasilar artery anastomosis, a rare congenital cerebrovascular variation [12]. The neurosurgeon should pay more attention to the presence of persistent cervical-basilar artery anastomosis to avoid damage to vascular anastomosis, thereby avoiding severe cerebral ischemia or infarction during surgery. 
Citation: Shi S (2017) Arterial Vascular Variation of the Head and Neck and Its Clinical Significance. J Neurol Neurophysiol 8: 442. doi: 10.4172/2155-9562.1000442

Page 2 of 2

The brain's vascular supply typically has a relatively generous collateralization, which is provided by the various anastomoses between the carotid and vertebrobasilar circulations and those between the left and right sides of the intracranial circulations in the circle of Willis (CoW) [13]. However, the author of this paper previously found an extremely rare variation of the ICA and vertebrobasilar artery, together with several other vascular variations in the head and neck [14]. The patient's head and neck CTA revealed the following rare vascular variations: (1) The bilateral CCAs share the common trunk issuing from the convex aortic arch; bilateral vertebral arteries became larger than the corresponding ICA; and the bilateral ICAs directly continued to the ophthalmic artery (OphA). (2) The ICA and vertebrobasilar artery were independent of each other without interconnected communicating branches; more importantly, the brain blood supply was entirely hosted by the vertebrobasilar artery.

In conclusion, the arterial vascular variations of the head and neck are diverse and inherent. Because of the compensatory effect of the body, most of these variations have no obvious influence on blood supply, which will accompany the patients for a lifetime. Multislice computed tomography angiography, MRA and ultrasound can noninvasively detect and evaluate the vascular anatomical variations of the head and neck in vivo, and these should be performed preoperatively. They can provide an accurate guidance for the neurosurgeon regarding the location, the type and the running course of vascular variations and help precise and safe preoperative planning.

\section{References}

1. Tsai IC, Tzeng WS, Lee T (2007) Vertebral and carotid artery anomalies in patients with aberrant right subclavian arteries. Pediatr Radiol 37: 1007-1012.

2. Bhatia K, Ghabrie MN, Henneberg M (2005) Anatomical variations in the branches of the human aortic arch: A recent study of a South Australian population. Folia Morphol (Warsz) 64: 217-223.
3. Chaturvedi S, Lukovits T, Chen W (1999) Ischemia in the territory of a hypoplastic vertebraobasilar system. Neurology 52: 980-983.

4. Sim E, Vaccaro AR, Berzlanovich A (2001) Fenestraion of the extracranial vertebral artery: Review of the literature. Spine 26: 139-142.

5. Miyazaki S, Kamata K, Yamaura A (1981) Multiple aneurysms of the vertebrabasilar system associated with fenestration of the vertebral artery. Surg Neurol 15: 192-195.

6. Cvetko E (2014) Concurrence of bilateral kinking of the extracranial part of the internal carotid artery with coiling and tortuosity of the external carotid artery - A case report. Rom J Morphol Embryol 55: 433-435.

7. Gartrell BC, Kennedy TA, Gubbels SP (2012) Bilateral duplicated internal carotid arteries presenting as middle ear masses: A case report and review of the literature. Ann Otol Rhinol Larynqol 121: 521-524.

8. Kwon WK, Park KJ, Park DH (2013) Ruptured saccular aneurysm arising from fenestrated proximal anterior cerebral artery: Case report and literature review. J Korean Neurosurg Soc 53: 293-296.

9. Uchino A, NomiyamaK, TakaseY (2006) Anterior cerebral artery variations detected by MR angiography. Neuroradiology 48: 647-652.

10. Uchino A, Ito S, Kurita H (2016) Duplicated middle cerebral artery arising from the origin of the hyperplastic anterior choroidal artery that mimicked aneurysm on routine MR angiography. Neuroradiol J 29: 106-109.

11. Lochner P, Golaszewski S, Caleri F (2011) Posterior circulation ischemia in patients with fetal-type circle of Willis and hypoplastic vertebrobasilar system. Neurol Sc 32: 1143-1146.

12. Caldemeyer KS, Carrico JB, Mathews VP (1998) The radiology and embryology of anomalous arteries of the head and neck. AJR Am J Roentgenol 170: 197-203.

13. Drummond JC, Lee RR, Howell JP (2012) Focal cerebral ischemia after surgery in the "beach chair"position: The role of a congenital variation of circle of Willis anatomy. Anesth Analq 114: 1301-1303.

14. Shi SK (2016) An unusual variation of carotid-vertebrobasilararteries: An anatomical case report. Neuroradiol J 29: 277-279. 